Bull. Austral. Math. Soc.

$47 \mathrm{H} 04,47 \mathrm{н} 10,54 \mathrm{H} 10,54 \mathrm{H} 25,55 \mathrm{M} 20,58 \mathrm{C} 60$

VOL. 58 (1998) [469-478]

\title{
FIXED POINTS OF UPPER SEMICONTINUOUS MAPPINGS IN LOCALLY $G$-CONVEX SPACES
}

\author{
GeORge XIAN-Zhi YuAN
}

\begin{abstract}
In this paper a new fixed point theorem for upper semicontinuous set-valued mappings with closed acyclic values is established in the setting of an abstract convex structure - called a locally $G$-convex space, which generalises usual convexity such as locally convex $H$-spaces, locally convex spaces (locally $H$-convex spaces), hyperconvex metric spaces and locally convex topological spaces. Our fixed point theorem includes corresponding Fan-Glicksberg type fixed point theorems in locally convex $H$-spaces, locally convex spaces, hyperconvex metric space and locally convex spaces in the existing literature as special cases.
\end{abstract}

\section{INTRODUCTION}

In nonlinear functional analysis, there are two basic types of topological fixed point theorems. The first one is the so-called Browder-Fan fixed point theorem (for example, see Browder [5] or its equivalent form in Fan [8]) which says that a set-valued selfmapping defined in a compact convex subset of a Hausdorff topological vector spaces has at least one fixed point if the set-valued mapping has open inverse values. The second type is the so-called Fan-Glicksberg fixed point theorem (for example, see Fan [7] or Glicksberg [9]) which says that an upper semicontinuous set-valued self-mapping defined in a compact convex subset of a Hausdorff locally topological vector space has at least one fixed point. The celebrated Fan-Glicksberg fixed point theorem is so general since it includes many fixed point theorems such as the Kakutani fixed point theorem for upper semicontinuous set-valued in Euclidean spaces, the Tychonoff, Schauder, Bohnenlus-Karlin and Brouwder, and many other fixed point theorems for continuous (single-valued) mappings in locally convex topological vector spaces, normed spaces, Banach spaces and Euclidean spaces as special cases. Fixed point theory in nonlinear analysis has been extensively studied and developed this century and its importance in nonlinear analysis and the essential relationships with other problems in nonlinear analysis can be found from Zeidler's books [31, Vol. I and Chapter 77 in Vol. IV] (and see also Goebel and Kirk's book [10] from the viewpoint of metric spaces for

Received 30th March, 1998

This project is supported in part by the Australian Research Council. The author thanks Professors E. Tarafdar, W.A. Kirk, S. Park and P. Watson for their helpful discussions and comments.

Copyright Clearance Centre, Inc. Serial-fee code: 0004-9729/98 \$A2.00+0.00. 
the study of fixed point theory and related applications in nonlinear analysis). However, we should mention that Schauder's conjecture raised in 1930's in fixed point theory is still unsolved (see Granas [13] and Dugundji and Granas [6] for more details); this asks if a (single-valued) continuous self-mapping defined in a compact and convex subset of a Hausdorff topological vector space has any fixed point.

On the other hand, as many problems in nonlinear analysis (in particular, in the study of economic models and game theory) can be formulated as equivalent to the existence of fixed points for a (typically, upper semicontinuous) set-valued mapping in the setting of spaces which, no longer, have linear structures such as locally convex topological vector spaces enjoy, it is necessary build some new theory for Fan-Glicksberg type fixed point theorems which can cover spaces which are not linear. In this direction, there are a few papers (for example, see Horvath [14, 15], Khamsi [17], Kirk and Shin [18], Park [20, 23], Park and Kim [23], Sine [24, 25], Tarafdar [28], Tarafdar and Watson [29], Wu [30] and others) which try to extend the Fan-Glicksberg fixed point theorem to the setting of general abstract convexity notions. However, all results so far obtained in this direction need to assume either both the lower and upper continuity of the set-valued mappings (see Tarafdar [28]) or that the underlying spaces are a hyperconvex metric space [1], or be a locally convex $H$-space in [30], or a locally convex space (called also, locally $H$-convex spaces by Tarafdar [28]) in Horvath [15].

The aim of this paper is to establish a Fan-Glicksberg type fixed point theorem for upper semicontinuous set-valued mappings with non-empty closed and acyclic (for example, contractible) values in the setting of the so-called locally $G$-convex spaces, which contain all locally convex $H$-spaces, locally convex spaces, hyperconvex metric space, and in particular, locally convex topological spaces as special cases. Thus our fixed point theorem shows that the celebrated Fan-Glicksberg type fixed point theorem holds in locally $G$-convex spaces, specially for locally convex $H$-spaces and locally $H$ convex spaces, hyperconvex metric spaces, which shows that the Fan-Glicksberg type fixed point theorem holds in hyperconvex metric spaces (see, for example, the corresponding fixed point theorems given by Kirk and Shin $[18$, p.180] under the assumption of continuity for set-valued mappings).

Throughout this paper, we denote by $\mathcal{F}(X)$ and $2^{X}$ the family of all non-empty finite subsets of $X$ and the family of all subsets of $X$ and we denote by $|A|$ the cardinal number of the set $A$. All topological spaces are assumed to be Hausdorff unless specified otherwise, for the simplicity of our discussion.

In the last few years, many papers have been devoted to proving the existence of fixed points for set-valued mappings in nonlinear analysis without traditional linear structures. As a result, several generalisations of convexity notions such as hyperconvex metric spaces, $H$-spaces, $G$-convex spaces have been introduced and studied by 
Aronszajn and Panitchpakdi [1], Bardaro and Ceppitelli [3], Bielawaski [4], Horvath $[14,15,16]$, Park $[20,21]$, Park and Kim [23], Tarafadr [28], Tarafdar and Watson [29], Wu [30] and others. Recently, in order to cover general economic models without linear convex structures, Park and Kim [23] introduced another abstract convexity notion-called a G-convex space, which includes many abstract convexity notions such as $H$-convex spaces as special cases. Formally we recall the following definition which was essentially given by Park and Kim [23].

Definition 1.1: A generalised convex space, or say, a $G$-convex space $(X, D ; \Gamma)$ consists of a topological space $X$, a non-empty subset $D$ of $X$ and a function $\Gamma: \mathcal{F}(X) \rightarrow X \backslash\{\emptyset\}$ such that (1): for each $A \in \mathcal{F}(X)$ with $|A|=n+1$, there exists a continuous function $\phi_{A}: \Delta_{n} \rightarrow \Gamma(A)$ such that $\phi_{A}\left(\Delta_{J}\right) \subset \Gamma(J)$ for each $\emptyset \neq J \subset\{0,1, \ldots, n\}$, where $A=\left\{x_{0}, x_{1}, \ldots, x_{n}\right\}$, and $\Delta_{J}$ denotes the face of $\Delta_{n}$ corresponding to the sub-index of $J$ in $\{0,1,2, \ldots, n\}$; and (2): A subset $C$ of the $G$-convex space $(X, D ; \Gamma)$ is said to be $G$-convex if for each $A \in \mathcal{F}(D), \Gamma_{A} \subset C$ for all $A \subset C$.

For the convenience of our discussion, we also denote $\Gamma(A)$ by $\Gamma_{A}$ or $\Gamma_{N}$ if there is no confusion for $A=\left\{x_{0}, x_{1}, x_{2}, \ldots, x_{n}\right\} \in \mathcal{F}(X)$, where $N$ is the set of all indices for the set $A$, that is, $N=\{0,1,2, \ldots, n\}$. A space $X$ is said to have a $G$-convex structure if and only of $X$ is a $G$-convex space. By Theorem 1 of Horvath [15], it is clear that the notion of $G$-convex spaces includes corresponding $H$-spaces (see its definition below) as a special class, which, in turn, includes topological vector spaces as special cases. In order to establish our fixed point theorem, we now introduce another notion called locally $G$-convex which include locally $H$-convex spaces, hyperconvex metric spaces (their definitions will be given shortly), and locally convex spaces as special cases.

Definition 1.2: A $G$-convex $X$ is said to be a locally $G$-convex space if $X$ is a uniform topological space with uniformity $\mathcal{U}$ which has an open base $\beta:=\left\{V_{i}: i \in I\right\}$ of symmetric entourages such that for each $V \in \beta$, the set $V(x):=\{y \in X:(y, x) \in V\}$ is a $G$-convex set for each $x \in X$.

\section{Fixed Point Theorems in Locally $G$-Convex Spaces}

In this section, we shall establish fixed point theorem for upper semicontinuous setvalued mappings with non-empty closed and acyclic values in locally $G$-convex spaces.

Recall that a non-empty space is said to be acyclic if all of its reduced Čech homology groups over the rationals vanish. In particular, any contractible space is acyclic, and thus any non-empty convex or star-shaped set is acyclic. Let $X$ and $Y$ be two topological spaces. We recall that a set-valued mapping $F: X \rightarrow 2^{Y}$ is said to have transfer open inversed values, if $X=\bigcup_{y \in Y} \operatorname{int}\left(F^{-1}(y)\right)$, where $\operatorname{int}\left(F^{-1}(y)\right)$ denotes the 
relative interior of the set $F^{-1}(y):=\{x \in X: y \in F(x)\}$ for each $y \in Y$ in $X$; and $F$ is said to be upper semicontinuous if the set $\{x \in X: F(x) \subset U\}$ is open for each open set $U$ in $Y$. When $Y$ is a $G$-convex space, the mapping $T: X \rightarrow 2^{Y}$ is said to have $G$-convex values if $T(x)$ is a $G$-convex set for each $x \in X$.

We first have the following selection result for a set-valued mapping with transfer open inversed values, which includes the corresponding result from Tarafdar [27, Lemma 2.2] as a special case.

LEMMA 2.1. Let $Y$ be a compact topological space and $X$ be a $G$-convex space. Suppose $T: Y \rightarrow 2^{X} \backslash\{\emptyset\}$ is an open inverse valued mapping with $G$-convex values. Then there exist some positive integer $n \in N$ and two single-valued continuous mappings $\phi: \Delta_{n} \rightarrow X$ and $\psi: Y \rightarrow \Delta_{n}$ such that the mapping $f: Y \rightarrow X$ defined by $f(y):=\phi(\psi(y))$ for each $x \in Y$ is a continuous selection of $T$, that is, $f(y) \in T(y)$ for all $y \in Y$, where $\Delta_{n}$ is the standard $n$-dimensional simplex with vertices $e_{0}, e_{1}, \ldots, e_{n}$.

Proof: Since $Y$ is compact and $T$ has open inversed values, it follows that $Y$ has a finite open cover $\left\{O_{i}: i=0,1, \ldots, n\right\}$, where $O_{i} \subset \operatorname{int}\left(F^{-1}\left(x_{i}\right)\right)$ for some $x_{i} \in X$. Let $A=\left\{x_{0}, x_{1}, \ldots, x_{n}\right\}$. Suppose $\left\{\psi_{i}\right\}_{i=0}^{n}$ is a partition of unity corresponding to the finite covering $\left\{O_{i}: i=0,1, \ldots, n\right\}$, that is, $\psi_{i}(y) \geq 0$ and $\Sigma_{i} \psi_{i}(y)=1$ and if $\psi_{i}(y)>0$, then $y \in O_{i}$. Set $J(y):=\left\{i \in I: \psi_{i}(y)>0\right\}$ for each $y \in Y$. Then for each $i \in J(y)$, it follows that $y \in O_{i} \subset T^{-1}\left(x_{i}\right)$, so that $x_{i} \in T(y)$. This means $A \cap T(x) \neq \emptyset$ for all $x \in X$. Secondly, the mapping $\psi: Y \rightarrow \Delta_{n}$ defined by $\psi(y):=\sum_{i=0}^{n} \psi_{i}(y) e_{i}$ for each $y \in Y$ is continuous. Since $X$ is a $G$-convex space, there exists a continuous mapping $\phi: \Delta_{n} \rightarrow \Gamma_{A}$ such that $\phi\left(\Delta_{J}\right) \subset \Gamma_{J}$ for each non-empty finite subset $J$ in $\{0,1, \ldots, n\}$. Then the mapping $f: Y \rightarrow X$ defined by $f(y):=\phi(\psi(y))$ for each $y \in Y$, is continuous. Since $T(y)$ is $G$-convex, it follows that $f(y):=\phi(\psi(y)) \subset \phi\left(\Delta_{J(y)}\right) \subset \Gamma_{J(y)} \subset T(y)$ for each $y \in Y\left(\right.$ as $\left.\Gamma_{J(y)} \subset T(y)\right)$, that is, $f(y) \in T(y)$ for each $y \in Y$, and this completes the proof.

Before establishing our fixed point theorem, we also recall the following result which is Lemma 2.1 of Park et al [22] from the Lefschetz-type fixed point theorem for composites of acyclic maps due to Gorniewicz and Granas [11] and [12].

LEMMA 2.2. Let $\Delta_{n}$ be a standard n-dimensional simplex with the Euclidean topology and $Y$ be a compact topological space. Let $\psi: Y \rightarrow \Delta_{n}$ be a single-valued continuous mapping and $T: \Delta_{n} \rightarrow 2^{Y}$ be a set-valued upper semicontinuous mapping with non-empty compact acyclic (for example, contractible) values. Then there exists $x_{0} \in \Delta_{n}$ such that $x_{0} \in \psi \circ T\left(x_{0}\right)$, where $\psi \circ T$ denotes the composition of the mapping $T$ with $\psi$.

We now prove the following fixed point theorem in locally $G$-convex spaces. This is a generalisation of the Fan-Glicksberg type fixed point theorems for upper semicontin- 
uous set-valued mappings with non-empty closed acyclic values given in several places (for example, see Kirk and Shin [18], Park [20, 21, 22, 23], Tarafdar [27, 28, 29], Wu $[30]$ and others in locally convex spaces).

THEOREM 2.1. Let $X$ be a compact locally $G$-convex space and $F: X \rightarrow 2^{X}$ be an upper semicontinuous set-valued mappings with non-empty closed acyclic values. Then $F$ has a fixed point, that is, there exists $x_{0} \in X$ such that $x_{0} \in F\left(x_{0}\right)$.

Proof: Since $\left(X, \Gamma_{A}\right)$ is a locally Hausdorff $G$-convex space, $X$ has a uniform structure $\mathcal{U}$ and without loss of generality, suppose $\mathcal{V}:=\left\{V_{i}: i \in I\right\}$ is a symmetric and open base family for the uniform structure $\mathcal{U}$. Then for each base $V_{i}$ and $x \in$ $X$, the set $V_{i}(x):=\left\{y \in X:(y, x) \in V_{i}\right\}=\left\{y \in X:(x, y) \in V_{i}\right\}$ is an open $G$-convex set, and $\bigcap_{i \in I} V_{i}=\Delta=\{(x, x): x \in X\}$. For each $V_{i}$, we define a setvalued mapping $T_{i}: X \rightarrow 2^{X}$ by $T_{i}(x):=\left\{y \in X:(y, x) \in V_{i}\right\}=V_{i}(x)$ for each $x \in X$. Then it is clear $T_{i}$ is a set-valued mapping from $X$ to $X$ with non-empty $G$-convex values and $\bigcup_{y \in X} \operatorname{int}\left(T_{i}^{-1}(y)\right) \subset X$ by the fact that for each $y \in X$, the set $T_{i}^{-1}(y)=\left\{x \in X:(x, y) \in V_{i}\right\}$ is open in $X$, so that $\operatorname{int}\left(T_{i}^{-1}(y)\right)=T_{i}^{-1}(y)$. By Lemma 2.1, there exist some positive integer $n \in N$ and two (single-valued) continuous mappings $g_{i}: \Delta_{n} \rightarrow X$ and $\phi_{i}: X \rightarrow \Delta_{n}$ such that $f_{i}(x):=g_{i}\left(\phi_{i}(x)\right) \in T_{i}(x)$ for each $x \in X$, where $\Delta_{n}$ is a standard $n$-dimensional simplex. Now we define another set-valued mapping $P_{i}: \Delta_{n} \rightarrow 2^{X}$ by $P_{i}(x):=F\left(g_{i}(x)\right)$ for each $x \in \Delta_{n}$. Then $P_{i}$ is an upper semicontinuous set-valued mapping with non-empty closed and acyclic values. By Lemma 2.2, the mapping $\phi_{i} \circ P_{i}: \Delta_{n} \rightarrow 2^{\Delta_{n}}$ has a fixed point, that is, there exists $u_{v_{i}} \in \Delta_{n}$ such that $u_{v_{i}} \in \phi_{i}\left(P_{i}\left(u_{v_{i}}\right)\right)$. Then there exists $x_{v_{i}} \in P_{i}\left(u_{v_{i}}\right)=$ $F\left(g_{i}\left(u_{v_{i}}\right)\right) \subset X$ and $u_{v_{i}}=\phi_{i}\left(x_{v_{i}}\right)$. Let $y_{v_{i}}=g_{i}\left(u_{v_{i}}\right)=g_{i}\left(\phi_{i}\left(x_{v_{i}}\right)\right)=f_{i}\left(x_{v_{i}}\right) \epsilon$ $T_{i}\left(x_{v_{i}}\right)$. Thus $x_{v_{i}} \in F\left(y_{v_{i}}\right)$, that is, $\left(x_{v_{i}}, y_{v_{i}}\right) \in \operatorname{Graph} F$ and $\left(x_{v_{i}}, y_{v_{i}}\right) \in V_{i}$, where Graph $F$ denotes the graph of the mapping $F$. Since $X \times X$ is compact, we may assume that $\left\{x_{v_{i}}, y_{v_{i}}\right\}_{i \in I}$ converges to $\left(x_{0}, y_{0}\right)$. Note that $F$ is an upper semicontinuous mapping with non-empty closed values, it follows that the graph Graph $F$ of $F$ is closed and thus $\left(x_{0}, y_{0}\right) \in \operatorname{Graph} F$, that is, $y_{0} \in F\left(x_{0}\right)$. In order to finish the proof, it suffices to show that $x_{0}=y_{0}$. Since $\left\{V_{i}: i \in I\right\}$ is an open symmetric base family for the uniform structure $\mathcal{U}$ and $\lim _{i \in I}\left(x_{v_{i}}, y_{v_{i}}\right)=\left(x_{0}, y_{0}\right)$, without loss of generality, there exists $V_{0} \in\left\{V_{i}: i \in I\right\}$ such that $\left(x_{v_{j}}, x_{0}\right) \in V_{j}$ and $\left(y_{v_{j}}, y_{0}\right) \in V_{j}$ for all $V_{j} \in\left\{V_{i}: i \in I\right\}$ with $V_{j} \subset V_{0}$. Now for any $U \in\left\{V_{i}: i \in I\right\}$ with $U \subset V_{0}$, let $V_{u} \in\left\{V_{i}: i \in I\right\}$ with $V_{u} \subset V_{0}$ be such that $V_{u} \circ V_{u} \circ V_{u} \subset U$. Since $\left(x_{v_{u}}, y_{v_{u}}\right) \in V_{u}$, it follows that $\left(x_{0}, y_{v_{u}}\right) \in V_{u} \circ V_{u}$ and thus $\left(x_{0}, y_{0}\right) \subset V_{u} \circ V_{u} \circ V_{u} \subset U$. Therefore we have show that $\left(x_{0}, y_{0}\right) \in U$ for all $U \in\left\{V_{i}: i \in I\right\}$ with $U \subset V_{0}$. Note that $\bigcap\left\{U: U \in \mathcal{V}\right.$, and $\left.U \subset V_{0}\right\}=\bigcap\left\{V_{i}: i \in I\right\}=\Delta$. This implies that $x_{0}=y_{0}$ and thus 
$x_{0}$ is a fixed point of $F$. This completes the proof.

For a given set $X$, we recall that a pair $\left(X,\left\{\Gamma_{A}\right\}\right)$ is said to be an $H$-space (called $c$-space by Horvath [15]) according to Bardaro and Ceppitelli [3] (motivated by earlier work of Horvath [14]) if $X$ is a topological space and $\left\{\Gamma_{A}\right\}_{A \in \mathcal{F}(X)}$ a given family of non-empty contractible subsets $\Gamma_{A}$ of $X$, indexed by $A \in \mathcal{F}(X)$, such that $\Gamma_{A} \subset F_{B}$ whenever $A \subset B$. Let $\left(X,\left\{\Gamma_{A}\right\}\right)$ be an $H$-space. A non-empty subset $D$ of $X$ is said to be $H$-convex (called an $F$-set by Horvath [15]) if $\Gamma_{A} \subset D$ for each $A \in \mathcal{F}(D)$

We also recall that an $H$-space is said to be a locally $H$-convex space in Tarafdar [28] (also called lower convex-space by Horvath [15]) if $X$ is a uniform topological space with uniformity $\mathcal{U}$ which has an open base $\beta:=\left\{V_{i}: i \in I\right\}$ of symmetric entourages such that for each $V \in \beta$, the set $V(S):=\{x \in X:(y, x) \in V$ for some $y \in S\}$ is an $H$-convex set for each $H$-convex set $S$ of $X$. From the notion of $H$-convex sets, it is clear that each $H$-convex set is also an $G$-convex set, but the converse is not true. Recently, Wu [30] introduced another notion called locally convex $H$-spaces which are a slight generalisation of Horvath's notion of locally convex-spaces and the concept of locally $H$-convexity given by Tarafdar [28] as follows.

Definition 2.1: An $H$-space $X$ is said to be a locally convex $H$-space if $X$ is a uniform topological space with uniformity $\mathcal{U}$ which has an open base $\beta:=\left\{V_{i}: i \in I\right\}$ of symmetric entourages such that for each $V \in \beta$, the set $V(x):=\{y \in X:(y, x) \in V\}$ is an $H$-convex set for each $x \in X$.

It is clear each locally convex $H$-space is a locally $G$-convex space. Note that if $X$ is a compact convex subset of a locally convex Hausdorff topological vector space $E$ and let $\Gamma_{A}:=\operatorname{co}(A)$ for each $A \in \mathcal{F}(X)$. Then $\left(X, \Gamma_{A}\right)$ is a locally convex $H$ space. Thus Theorem 2.1 is really a generalisation of the celebrated Fan-Glicksberg fixed point theorem for upper semicontinuous set-valued mappings with non-empty closed and acyclic (for example, contractible) values in both locally convex $H$-spaces, locally convex spaces (locally $H$-convex spaces) and locally convex topological vector spaces. Theorem 2.1 shows that Theorem 2.1 of Tarafdar [28] is true when the mapping $F$ is only upper semicontinous. Theorem 2.1 also extends Theorem 3.2 of Tarafdar and Watson [29] and the corresponding Fan-Glicksberg type fixed point theorems given by $\mathrm{Wu}[\mathbf{3 0}]$ to locally $G$-convex spaces which include locally $H$-convex space and locally convex $H$-spaces as special classes. For example, as special cases of Theorem 2.1 we have the following generalisations of Fan-Glicksberg type fixed point theorems in both locally convex $H$-spaces and locally convex Hausdorff topological vector spaces (for example, see also Wu [30], Park $[20,21,22,23]$ and related references there, Fan [7] and Glicksberg [9] and others).

COROLlary 2.2. Let $X$ be a compact locally convex $H$-space and $F: X \rightarrow 2^{X}$ 
be an upper semicontinuous set-valued mapping with non-empty closed acyclic values. Then $F$ has a fixed point $x_{0} \in X$.

CoRollary 2.3. Let $X$ be a compact convex subset of a Hausdorff locally convex topological vector space and $F: X \rightarrow 2^{X}$ be an upper semicontinuous setvalued mapping with non-empty closed acyclic (for example, convex) values. Then $F$ has a fixed point $x_{0} \in X$.

COROLlary 2.4. Let $X$ be a compact convex subset of a Hausdorff locally convex topological vector space and $F: X \rightarrow 2^{X}$ be an upper semicontinuous set-valued mapping with non-empty closed convex values. Then $F$ has a fixed point $x_{0} \in X$.

In order to discuss the Fan-Glicksberg fixed point theorem in hyperconvex metric spaces for upper semicontinuous set-valued mappings, we recall the following definition of a hyperconvex metric space due to Aronszajn and Panitchpakdi [1].

Definition 2.2: A metric space $(X, d)$ is said to be a hyperconvex space if for any collection of points $\left\{x_{\alpha}\right\}$ of $X$ and any collection $\left\{r_{\alpha}\right\}$ of non-negative real numbers with $d\left(x_{\alpha}, x_{\beta}\right) \leq r_{\alpha}+r_{\beta}$, we have $\bigcap_{\alpha} B\left(x_{\alpha}, r_{\alpha}\right) \neq \emptyset$, where $B(x, r)$ denotes the closed ball centred at $x \in X$ with the radius $r$.

The relationship between hyperconvex metric spaces and nonexpansive mappings is an important one as shown independently by the work of Sine [25] and Soardi [26]. Hyperconvex metric spaces have been used widely and many interesting results for nonexpansive mappings have been established in the framework of hyperconvex structures, for example, see Baillon [2], Goebel and Kirk [10], Khamsi [17], Since [24, 25] and others. Since a hyperconvex space is a nonexpansive retract of any metric space in which it is embedded (for example, see [1], Lacey [19] or Khamsi [17]) and since any metric space can be embedded in a Banach space, it follows that any hperconvex metric space is contractible. Note that if $\left\{B\left(y_{i}, r_{i}\right)\right\}_{i \in I}$ is any collection of closed balls in a hyperconvex space, then $\bigcap_{i \in I} B\left(y_{i}, r_{i}\right)$ itself is a hyperconvex space. Therefore for a hyperconvex space $(Y, d)$, the set $\bigcap_{i \in I} B\left(y_{i}, r_{i}\right)$ is contractible, or empty. For any nonempty finite subset $A \in \mathcal{F}(Y)$, the admissible set of $A$ (called the ball hull of $A$ by Sine in [25]) defined by $\Gamma_{A}:=\bigcap\{B(y, r): A \subset B(y, r)\}$ defines an $H$-structure in $Y$ and thus each hyperconvex metric space $(Y, d)$ is an $H$-space with the $H$-structure $\Gamma_{A}$ given by the ball hull.

If a locally $H$-convex space is a metric space, an equivalent definition has been given by Horvath [15] (where it is called a locally convex metric space) as follows.

Definition 2.3: Let the metric space $\left(Y, \Gamma_{A}\right)$ be an $H$-space with the metric $d$, where $\Gamma_{A}$ is defined as above for each $A \in \mathcal{F}(Y)$. Then $Y$ is said to be a locally $H$-convex space if for each $r>0$, the set $\{y \in Y: d(y, E)<r\}$ is $H$-convex for each 
$H$-convex set $E$ in $Y$, and also all open balls are $H$-convex.

Each hyperconvex metric space is a complete local $H$-convex space as shown by the following result, which was first given by Horvath [16]; and for the convenience of readers, we include his proof here.

LEMMA 2.3. Each hyperconvex metric space $(Y, d)$ is a complete locally metrically convex $H$-space (and thus $(Y, d)$ is a locally $G$-convex space).

Proof: By following Horvath [16], for each $y \in Y, \Gamma_{\{y\}}=\{y\}$ which, of course, is an $H$-convex set. We have to show that for any $H$-convex set $E$ and any $r>0$, the set $\{y \in Y: d(y, e)<r\}$ is an $H$-convex set. Let $y_{0}, \ldots, y_{n} \in Y$ such that $d\left(y_{i}, E\right)<r$ and $y_{0} \in \Gamma_{\left\{y_{1}, \ldots, y_{n}\right\}}$, that is, $y_{0}$ belongs to any closed ball containing $\left\{y_{1}, \ldots, y_{n}\right\}$. We want to show that $d\left(y_{0}, E\right)<r$. Take any points $\widehat{y}_{1}, \ldots, \widehat{y}_{n} \in E$ such that $d\left(y_{i}, \widehat{y}_{i}\right)<r$ for $i=1, \ldots, n$. Since $\Gamma_{\left\{\widehat{y}_{1}, \ldots, \widehat{y}_{n}\right\}} \subset E$, it suffices to show that $d\left(y_{0}, \Gamma_{\left\{\hat{y}_{1}, \ldots, \hat{y}_{n}\right\}}\right)<r$. By the definition of $\Gamma_{\left\{\hat{y}_{1}, \ldots, \hat{y}_{n}\right\}}, \Gamma_{\left\{\hat{y}_{1}, \ldots, \hat{y}_{n}\right\}}=\bigcap_{i \in I} B\left(u_{i}, r_{i}\right)$, where $\left(u_{i}, r_{i}\right) \in Y \times R_{+}$. Choose $r^{\prime} \in(0, r)$ such that $d\left(y_{i}, \widehat{y}_{i}\right)<r^{\prime}$ for $i=1, \ldots, n$. As $\left\{\widehat{y}_{1}, \ldots, \widehat{y}_{n}\right\} \subset B_{i}\left(u_{i}, r_{i}\right)$ for $i \in I$, it follows that $\left\{y_{1}, \ldots, y_{n}\right\} \subset B\left(u_{i}, r_{i}+r\right)$ for $i \in I$ and $y_{0} \in \bigcap_{i \in I} B\left(u_{i}, r_{i}+r^{\prime}\right)$. By Sine's Lemma in [25, p.864], there exists a retraction $R: \bigcap_{i \in I} B\left(u_{i}, r_{i}+r^{\prime}\right) \rightarrow \bigcap_{i \in I} B\left(u_{i}, r_{i}\right)$ such that $d(y, R(y)) \leq r^{\prime}$ for any $y \in \bigcap_{i \in I} B\left(u_{i}, r_{i}+r^{\prime}\right)$. Then $R\left(y_{0}\right) \in \Gamma_{\left\{\hat{y}_{1} \ldots, \hat{y}_{n}\right\}}$ and $d\left(y_{0}, R\left(y_{0}\right)\right) \leq r^{\prime}<r$. This completes the proof.

By Lemma 2.3 and Theorem 2.1, we have the following fixed point result in hyperconvex metric spaces.

Corollary 2.5. Let $X$ be a compact admissible set in a hyperconvex metric space $M$ and $F: X \rightarrow 2^{X}$ be an upper semicontinuous set-valued mapping with non-empty closed acyclic values. Then $F$ has a fixed point $x_{0} \in X$.

In their paper [18, p.180], Kirk and Shin prove a fixed point theorem for continuous set-valued mappings and they ask whether their result remains true under the assumption that the mapping is upper semicontinuous rather than continuous. Corollary 2.5 shows that their result holds for upper semicontinuous set-valued mappings.

\section{REFERENCES}

[1] N. Aronszajn and P. Panitchpakdi, 'Extensions of uniformly continuous transformations and hyperconvex metric spaces', Pacific J. Math. 6 (1956), 405-439.

[2] J.B. Baillon, 'Nonexpansive mappings and hyperconvex spaces', in Fixed point theory and its applications, (R. F. Brown, Editor) (Contemp. Maths. 72, Amer. Math. Soc., Providence, R.I., 1988), pp. 11-19. 
[3] C. Bardaro and R. Ceppitelli, 'Some further generalization of Knaster - Kuratowski - Mazurkiewicz theorem and minimax inequalities', J. Math. Anal. Appl. 132 (1988), 484-490.

[4] R. Bielawaski, 'Simplicial convexity and its applications', J. Math. Anal. Appl. 127 (1987), 155-171.

[5] F.E. Browder, 'The fixed point theory of multivalued mappings in topological vector spaces', Math. Ann. 177 (1968), 283-301.

[6] J. Dugundji and A. Granas, Fixed point theory 1 (PWN, Warszawa, 1982).

[7] K. Fan, 'Fixed points and minimax theorems in locally convex topological linear spaces', Proc. Nat. Acad. Sci. U.S.A. 38 (1952), 131-136.

[8] K. Fan, 'A generalization of Tychonoff's fixed point theorem', Math. Ann. 142 (1961), 305-310.

[9] I.L. Glicksberg, 'A further generalization of the Kakutani fixed point theorem with applications to Nash equilibrium points', Proc. Amer. Math. Soc. 3 (1952), 170-174.

[10] K. Goebel and W.A. Kirk, Fixed point theory in metric spaces (Cambridge University Press, Cambridge, 1990).

[11] L. Gorniewicz and A. Granas, 'Some general theorems in coincidence theory I', J. Math. Pure Appl. 60 (1981), 361-373.

[12] L. Gorniewicz and A. Granas, 'Topology of morphisms and fixed point problems for set-valued maps', in Fixed point theory and applications, (M. A. Thera and J. B. Baillon, Editors) (Longman Sci. Tech., Essex, 1991), pp. 173-191.

[13] A. Granas, 'KKM-maps and their applications to nonlinear problems', in The Scottish Book: Mathematic from the Scottish Cafe, (R. Daniel Mauldin, Editor) (Boston, 1982), pp. $45-61$.

[14] C. Horvath, 'Some results on multivalued mappings and inequalities without convexity', in Nonlinear and convex analysis, (B.L. Lin and S. Simons, Editors) (Marcel Dekker, New York, 1987), pp. 96-106.

[15] C. Horvath, 'Contractiblity and generalized convexity', J. Math. Anal. Appl. 156 (1991), 341-357.

[16] C. Horvath, 'Extension and selection theorems in topological spaces with a generalized convexity structure', Ann. Fac. Sci. Toulouse Math. 2 (1993), 253-269.

[17] M.A. Khamsi, 'KKM and Ky Fan theorems in hyperconvex metric spaces', J. Math. Anal. Appl. 204 (1996), 298-306.

[18] W.A. Kirk and S.S. Shin, 'Fixed point theorems in hyperconvex spaces', Houston J. Math. 23 (1997), 175-187.

[19] H. E. Lacey, The isometric theory of classical Banach Spaces (Springer Verlag, Berlin, Heidelberg, New York, 1974).

[20] S. Park, 'Some coincidence theorems on acyclic multifunctions and applications to KKM theory', in Fixed point theory and applications, (K.K. Tan, Editor) (World Scientific, Singapore, 1992), pp. 248-278.

[21] S. Park, 'Fixed point theorems in hyperconvex metric spaces', Nonlinear Anal. (1998) (to appear). 
[22] S. Park, J.S. Bae and H.K. Kang, 'Geometric properties, minimax inequalities and fixed point theorems on convex spaces', Proc. Amer. Math. Soc. 121 (1994), 429-439.

[23] S. Park and H. Kim, 'Admissible classes of multifunctions on generalized convex spaces', Proc. Coll. Nat. Sci. SNU 18 (1993), 1-21.

[24] R.C. Sine, 'Hyperconvexity and nonexpansive multifunctions', Trans. Amer. Math. Soc. 315 (1989), 755-767.

[25] R.C. Sine, 'Hyperconvexity and approximate fixed points', Nonlinear Anal. 13 (1989), 863-869.

[26] P.M. Soardi, 'Existence of fixed points of nonexpansinve mappings in certain Banach lattices', Proc. Amer. Math. Soc. 73 (1979), 25-29.

[27] E. Tarafdar, 'Fixed point theorems in $H$-spaces and equilibrium points of abstract economies', J. Austral. Math. Soc. (Ser. A) 53 (1992), 252-260.

[28] E. Tarafdar, 'Fixed point theorems in locally $H$-convex uniform spaces', Nonlinear. Anal. 29 (1997), 971-978.

[29] E. Tarafdar and P. Watson, 'Coincidence and the Fan-Glicksberg fixed point theorem in locally $H$-convex uniform spaces', The University of Queensland (1997).

[30] X. Wu, 'On Kakutani-Fan-Glicksberg fixed point theory and applications in $H$-spaces', J. Math. Anal. Appl. (to appear).

[31] E. Zeidler, Nonlinear functional analysis and its applications. Vol. I: Fixed point theorems and Vol. IV: Applications to mathematical physiscs (Springer Verlag, Berlin, Heidelberg, New York, 1985).

Department of Mathematics

The University of Queensland

Brisbabne Qld 4072

Australia

e-mail: xzy@maths.uq.edu.au 